\title{
HIGH GLUCOSE EXPOSURE PROMOTES PROLIFERATION AND IN VIVO NETWORK FORMATION OF ADIPOSE-TISSUE-DERIVED MICROVASCULAR FRAGMENTS
}

\author{
M.W. Laschke ${ }^{1, *}$, M.S. Seifert ${ }^{1}$, C. Scheuer ${ }^{1}$, E. Kontaxi ${ }^{1}$, W. Metzger $^{2}$ and M.D. Menger ${ }^{1}$ \\ ${ }^{1}$ Institute for Clinical and Experimental Surgery, Saarland University, 66421 Homburg/Saar, Germany \\ ${ }^{2}$ Department of Trauma, Hand and Reconstructive Surgery, Saarland University, \\ 66421 Homburg/Saar, Germany
}

\begin{abstract}
High glucose concentrations have been shown to activate endothelial cells and promote angiogenesis. In the present study, it was investigated whether high glucose concentrations could improve the vascularisation capacity of adipose-tissue-derived microvascular fragments (ad-MVF). Ad-MVF were isolated from the epididymal fat pads of donor mice and cultivated for $24 \mathrm{~h}$ in University of Wisconsin (UW) solution supplemented with vehicle or $30 \mathrm{mM}$ glucose. Protein expression, morphology, viability and proliferation of the cultivated ad-MVF were analysed by means of proteome profiler mouse angiogenesis array, scanning electron microscopy and immunohistochemistry. Additional cultivated ad-MVF were seeded on to collagenglycosaminoglycan scaffolds to study their in vivo vascularisation capacity in the dorsal skinfold chamber model by intravital fluorescence microscopy, histology and immunohistochemistry. In vitro, high glucose exposure changed the protein expression pattern of ad-MVF with endoglin, interleukin (IL)- $1 \beta$ and monocyte chemoattractant protein (MCP)-1 as the most up-regulated pro-angiogenic factors. Moreover, high glucose exposure induced the formation of nanopores in the ad-MVF wall. In addition, ad-MVF contained significantly larger numbers of proliferating endothelial and perivascular cells while exhibiting a comparable number of apoptotic cells when compared to vehicle-treated controls. In vivo, scaffolds seeded with high-glucose-exposed ad-MVF exhibited an improved vascularisation and tissue incorporation. These findings demonstrated that the exposure of cultivated ad-MVF to high glucose concentrations is a promising approach to improve their in vivo performance as vascularisation units for tissue engineering and regenerative medicine.
\end{abstract}

Keywords: Tissue engineering, microvascular fragments, glucose, hyperglycaemia, angiogenesis, vascularisation, scaffold.

*Address for correspondence: Matthias W. Laschke, MD, PhD, Institute for Clinical and Experimental Surgery, Saarland University, D-66421 Homburg/Saar, Germany.

Telephone number: +49 68411626554 Fax number: +4968411626553 Email: matthias.laschke@uks.eu

Copyright policy: This article is distributed in accordance with Creative Commons Attribution Licence (http://creativecommons.org/licenses/by-sa/4.0/).

\section{Introduction}

Adipose-tissue-derived microvascular fragments (ad-MVF) are arteriolar, capillary and venular vessel segments, which are isolated by short-term enzymatic digestion of fat samples (Laschke and Menger, 2015). Originally, these vessel segments have been used in physiology and angiogenesis research to study endothelial transport processes (Williams et al., 1981), interaction of endothelial cells with extracellular matrix compounds (Madri et al., 1983) or effects of cytokines and growth factors on microvascular network formation (Sato et al., 1990; Sato et al., 1993). More recently, ad-MVF have been incorporated into different scaffold materials to improve the vascularisation of implanted tissue constructs, such as epicardial patches (Shepherd et al., 2007) or bone and dermal skin substitutes (Frueh et al., 2017a; Laschke et al., 2012).

Ad-MVF bear several unique advantages, which make them attractive vascularisation units for tissue engineering and regenerative medicine. They can be isolated in large amounts quickly from fat samples (Frueh et al., 2017b). Due to their preserved vessel architecture and functionality after isolation, they rapidly reassemble into new blood-perfused microvascular networks after in vivo implantation (Später et al., 2018). Moreover, 
they contain mesenchymal stem cells within their physiological niche, which exhibit a high capacity for angiogenic, neurogenic, adipogenic and osteogenic differentiation (McDaniel et al., 2014). In addition, adMVFs can be pre-cultivated for up to $24 \mathrm{~h}$ prior to their in vivo application without losing their physiological vessel morphology (Laschke et al., 2019a). This offers the possibility of further improving their angiogenic activity by exposing them to different physical and biochemical stimuli, such as subnormothermic temperatures and pro-angiogenic factors (Karschnia et al., 2018; Laschke et al., 2019a; Laschke et al., 2019b).

The study hypothesis was that the in vitro exposure of cultivated ad-MVF to high glucose concentrations increases their in vivo microvascular-networkforming capacity. This assumption was based on the fact that hyperglycaemia in diabetic patients is typically associated with excessive angiogenesis in different organs, which, for example, causes diabetic retinopathy and nephropathy (Aouiss et al., 2019; Zent and Pozzi, 2007). In line with this finding, several in vitro studies have reported that highglucose-treated endothelial cells exhibit an increased proliferating, migrating and tube-forming activity due to the upregulation of angiogenic signalling pathways (Betts-Obregon et al., 2016; Fernando et al., 2018; Qiu et al., 2018; Shi et al., 2019).

To test the study hypothesis, ad-MVF isolated from epididymal fat pads of donor mice were exposed in vitro for $24 \mathrm{~h}$ to high glucose and their protein expression, morphology, viability and proliferation were analysed in comparison to vehicle-exposed controls. Moreover, collagen-glycosaminoglycan scaffolds seeded with high-glucose- and vehicleexposed ad-MVFs were implanted into dorsal skinfold chambers of recipient mice to study their in vivo vascularisation and incorporation.

\section{Materials and Methods}

\section{Ethical statement}

The study was approved by the local governmental animal protection committee (Landesamt für Verbraucherschutz, Abteilung C Lebensmittel- und Veterinärwesen, Saarbrücken, Germany; permission number: 29/2014). All experiments were conducted in accordance with the Directive 2010/63/EU and the NIH Guidelines for the Care and Use of Laboratory Animals (NIH Publication \#85-23 Rev. 1985).

\section{Animals}

For the isolation of ad-MVF, male C57BL/6 wild-type mice and C57BL/6-TgN(ACTB-EGFP)1Osb/J mice (age: 6-12 months; body weight: > $30 \mathrm{~g}$; Institute for Clinical and Experimental Surgery, Saarland University, Homburg/Saar, Germany) were used as fat donors. The latter ubiquitously express green fluorescent protein (GFP) (Okabe et al., 1997). Dorsal skinfold chambers were implanted into C57BL/6 wild-type mice (age: 3-5 months; body weight: 22$27 \mathrm{~g}$ ). Animals were housed in the animal facility of the Institute for Clinical and Experimental Surgery (Saarland University, Homburg/Saar, Germany) with access to tap water and standard pellet food ad libitum (Altromin, Lage, Germany).

\section{Isolation and cultivation of ad-MVF}

Ad-MVF were isolated from the epididymal fat pads of donor mice (Fig. 1a-c), as described previously in detail (Frueh et al., 2017b). In brief, the donor animals were anaesthetised by intraperitoneal injection of ketamine $(100 \mathrm{mg} / \mathrm{kg}$ body weight; Ursotamin, Serumwerk Bernburg AG, Bernburg, Germany) and xylazine (12 mg/kg body weight; Rompun, Bayer, Leverkusen, Germany), laparotomised and sacrificed by incision of the abdominal aorta. The epididymal fat pads were excised, mechanically minced and digested in collagenase NB4G $(0.5 \mathrm{U} / \mathrm{mL}$; Serva, Heidelberg, Germany) for 9 min while being stirred at $37^{\circ} \mathrm{C}$ under humidified atmospheric conditions. Subsequently, the enzyme was neutralised with phosphate-buffered saline (PBS) containing $20 \%$ foetal calf serum (FCS) and the suspension was filtered through a $500 \mu \mathrm{m}$ mesh (pluriSelect Life Science, Leipzig, Germany) to remove non-digested fat clots. Moreover, fat supernatants were carefully removed. The remaining ad-MVF were finally enriched up to a pellet size by centrifugation for $5 \mathrm{~min}$ at $120 \times g$.

The isolated ad-MVF were cultivated for $24 \mathrm{~h}$ under humidified conditions in $1 \%$ agarose-coated 24-well plates containing $4^{\circ} \mathrm{C}$ University of Wisconsin (UW) solution (Belzer UW Cold Storage Solution, Bridge to Life Ltd., Columbia, SC, USA). These culture conditions have previously been proven to be suitable for the xeno-free cultivation of ad-MVF, as it would be necessary in future clinical practice (Laschke et al., 2019b). The UW solution was supplemented with $30 \mathrm{mM}$ glucose (high glucose; Carl Roth, Karlsruhe, Germany) or vehicle (control; PBS). At the end of the $24 \mathrm{~h}$ cultivation period, the ad-MVF were washed in PBS prior to further analyses.

\section{Protein expression of ad-MVF}

The expression of 53 pro- and anti-angiogenic proteins was analysed in vehicle- and high-glucoseexposed ad-MVF by means of a proteome profiler mouse angiogenesis array kit (R\&D Systems, Wiesbaden, Germany), as described previously in detail (Laschke et al., 2018).

\section{Scaffold preparation and seeding}

Vehicle- and high-glucose-exposed ad-MVF were seeded on to clinically available collagenglycosaminoglycan scaffolds (Integra ${ }^{\circledR}$; Integra $\mathrm{GmbH}$, Ratingen, Germany) having a diameter of $3 \mathrm{~mm}$ (Fig. 1c). A detailed description of the scaffold preparation and seeding procedure is given in Laschke et al. (2019b). 

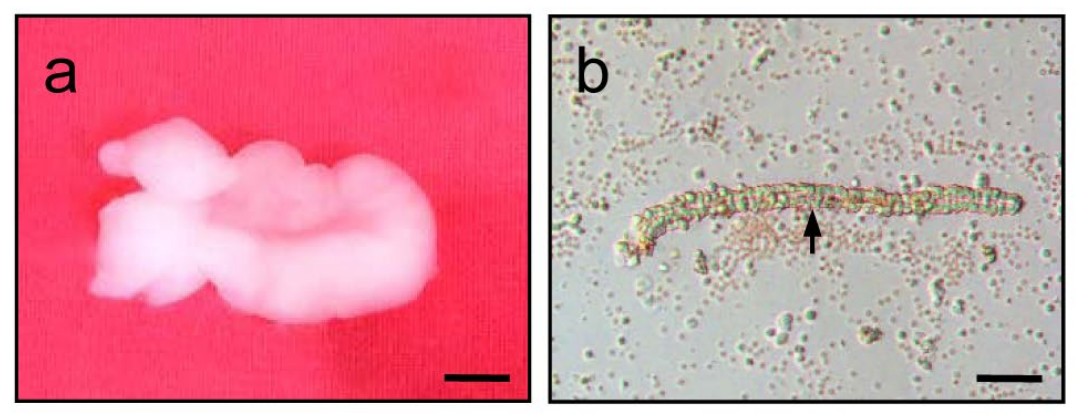

Fig. 1. Isolation of ad-MVF and experimental model. (a) Epididymal fat pad of a C57BL/6 donor mouse. Scale bar: $3.5 \mathrm{~mm}$. (b) Brightfield microscopy of an ad-MVF (arrow) directly after enzymatic isolation from the epididymal fat pad. Scale bar: $35 \mu \mathrm{m}$. (c) Schematic overview of the ad-MVF isolation and preparation

C
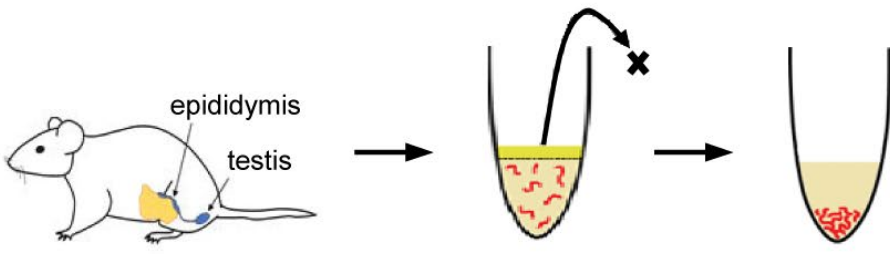

removal of fat supernatant

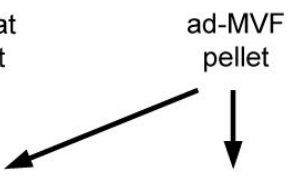

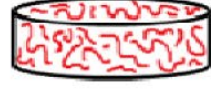

scaffold seeding
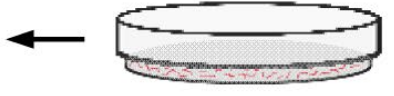

high glucose

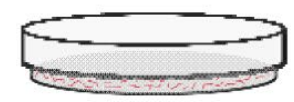

vehicle
$4{ }^{\circ} \mathrm{C}$ UW solution
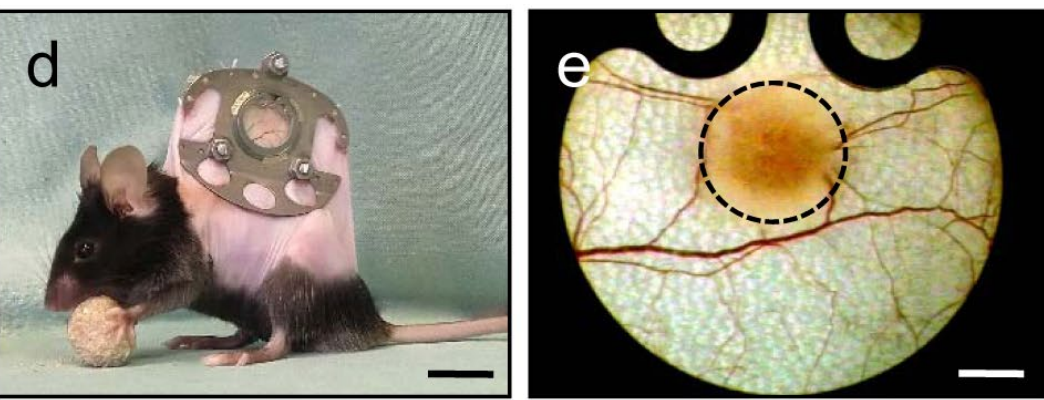
procedure. Ad-MVF were isolated by enzymatic digestion of the epididymal fat pads of donor mice. After the removal of fat supernatants, ad-MVF were enriched up to a pellet size and subsequently cultivated for $24 \mathrm{~h}$ in $4{ }^{\circ} \mathrm{C}$ UW solution containing high glucose or vehicle. Thereafter, the ad-MVF were seeded on to collagenglycosaminoglycan scaffolds for further in vitro and in vivo analyses. (d) C57BL/6 mouse with a dorsal skinfold chamber. Scale bar: $12.5 \mathrm{~mm}$. (e) Stereomicroscopy of the observation window of a dorsal skinfold chamber after the implantation of an ad-MVF-seeded collagen-glycosaminoglycan scaffold (borders marked by broken line). Scale bar: $1.5 \mathrm{~mm}$.

\section{Morphology of ad-MVF}

The morphology of vehicle- and high-glucose-exposed ad-MVF seeded on to collagen-glycosaminoglycan scaffolds was analysed by means of a FEI XL-30 ESEM FEG scanning electron microscopy device (FEI, Hillsboro, OR, USA). A detailed description of the sample preparation is given in Laschke et al. (2018).

\section{Dorsal skinfold chamber model and intravital fluorescence microscopy}

Vehicle- and high-glucose-exposed ad-MVF from $\mathrm{GFP}^{+}$donor mice were seeded on to collagenglycosaminoglycan scaffolds, which were implanted into dorsal skinfold chambers of $\mathrm{GFP}^{-}$recipient mice (Fig. 1d,e) and analysed by means of intravital fluorescence microscopy, as described previously in detail (Laschke et al., 2019a). For contrast enhancement by intravascular staining of plasma, $100 \mu \mathrm{L} 5 \%$ FITC-labelled dextran 150,000 (Sigma-Aldrich) were injected before each microscopy analysis into the retrobulbary plexus of the anaesthetised mice. The microscopy images were analysed with the computer-assisted off-line analysis system CapImage (Zeintl, Heidelberg, Germany). The vascularisation of the implants was assessed in 8 regions of interest (ROIs). Perfused ROIs (in percentage of all ROIs) were defined as areas containing either newly developed red blood cell (RBC)-perfused microvessels or reperfused $\mathrm{GFP}^{+}$ad-MVF. In addition, the functional microvessel density, i.e. the length of all RBCperfused microvessels per ROI given in $\mathrm{cm} / \mathrm{cm}^{2}$, as well as the diameter ( $\mathrm{D}$; given in $\mu \mathrm{m}$ ) and the centreline RBC velocity ( $v$; given in $\mu \mathrm{m} / \mathrm{s}$ ) of 30 randomly selected perfused microvessels within the implants were measured. The wall shear rate (y; given in $\mathrm{s}^{-1}$ ) of these vessels was then calculated with the formula: $\mathrm{y}=8 \times \mathrm{v} / \mathrm{D}$.

\section{Experimental protocol}

For in vitro analyses, ad-MVF were isolated from epididymal fat pads of $32 \mathrm{GFP}^{-}$donor mice. Isolated ad-MVF were divided into two equal parts and cultivated for $24 \mathrm{~h}$ in $4{ }^{\circ} \mathrm{C}$ UW solution, which was supplemented with vehicle or high 
Table 1. Expression of angiogenesis-related proteins (percentage of vehicle) in ad-MVF as assessed by a proteome profiler mouse angiogenesis array. ad-MVF were cultivated for $24 \mathrm{~h}$ in $4{ }^{\circ} \mathrm{C}$ UW solution supplemented with vehicle or high glucose.

\begin{tabular}{|c|c|}
\hline Protein & Expression (percentage of vehicle) \\
\hline \multicolumn{2}{|c|}{ Pro-angiogenic } \\
\hline Endoglin/CD105 & 203.2 \\
\hline IL-1 $\beta$ & 190.0 \\
\hline MCP-1/CCL2/JE & 184.5 \\
\hline HGF & 152.4 \\
\hline KC/CXCL1/CINC-1/GRO $\alpha$ & 148.9 \\
\hline EGF & 128.0 \\
\hline Osteopontin/OPN & 113.9 \\
\hline FGF basic/FGF-2 & 113.2 \\
\hline SDF-1/CXCL 12 & 110.7 \\
\hline Leptin/OB & 107.3 \\
\hline GM-CSF & 102.0 \\
\hline Angiogenin/ANG & 101.7 \\
\hline FGF acid/FGF-1/ECGF/HBGF-1 & 99.8 \\
\hline Amphiregulin/AR & 99.7 \\
\hline Angiopoietin-1/Ang-1 & 95.3 \\
\hline Coagulator factor III/Tissue factor/TF & 95.3 \\
\hline Endothelin-1/ET-1 & 94.4 \\
\hline DLL4 & 92.5 \\
\hline MMP-9 & 90.9 \\
\hline CXCL 16 & 90.7 \\
\hline NOV/CCN3/IGFBP-9 & 90.6 \\
\hline KGF/FGF-7 & 90.1 \\
\hline IL-1 & 89.9 \\
\hline IGFBP-2 & 89.7 \\
\hline IGFBP-1 & 87.4 \\
\hline VEGF B/VRF & 87.2 \\
\hline Cyr61/CCN1, IGFBP-10 & 83.7 \\
\hline HB-EGF & 83.6 \\
\hline VEGF/VPF & 82.3 \\
\hline PDGF-AA & 79.8 \\
\hline Fractalkine/CX3CL 1 & 79.3 \\
\hline Proliferin & 67.2 \\
\hline IL-10/CSIF & 67.0 \\
\hline MIP-1 $\alpha$ & 65.8 \\
\hline PD-ECGF & 64.7 \\
\hline MMP-3 & 64.7 \\
\hline PIGF-2 & 62.2 \\
\hline IGFBP-3 & 60.1 \\
\hline MMP-8 & 38.7 \\
\hline \multicolumn{2}{|c|}{ Anti-angiogenic } \\
\hline Endostatin/Collagen VIII & 148.8 \\
\hline Serpin E1/PAI-1 & 111.6 \\
\hline ADAMTS1/METH1 & 106.9 \\
\hline DPP IV/CD26 & 104.1 \\
\hline PDGF-AB/BB & 103.0 \\
\hline Platelet factor 4/CXCL4/PF4 & 102.6 \\
\hline Thrombospondin-2/TSP-2 & 83.8 \\
\hline Serpin F1/PEDF & 79.5 \\
\hline Pentraxin-3/PTX3/TSG-14 & 74.3 \\
\hline Prolactin/PRL & 66.5 \\
\hline TIMP-4 & 63.4 \\
\hline IP-10/CXCL 10 & 63.3 \\
\hline Angiopoietin-3/Ang-3 & 60.8 \\
\hline TIMP-1 & 42.6 \\
\hline
\end{tabular}


glucose. Immediately after cultivation, the protein expression of the ad-MVF was analysed by means of a proteome profiler mouse angiogenesis array kit ( $n=2$ per group). Additional ad-MVF were seeded on to collagen-glycosaminoglycan scaffolds to study their morphology by means of scanning electron microscopy ( $n=2$ per group) and their viability and proliferation by means of immunohistochemistry ( $n=4$ per group).

For in vivo analyses, ad-MVF were isolated from the epididymal fat pads of $20 \mathrm{GFP}^{+}$donor mice and cultivated as described above. Subsequently, they were seeded on to collagen-glycosaminoglycan scaffolds, which were implanted into the dorsal skinfold chambers of $18 \mathrm{GFP}^{-}$recipient mice (vehicle: $n=9$; high glucose: $n=9$ ). The scaffolds were analysed directly after implantation (day 0) and on days 3, 6, 10 and 14 by means of intravital fluorescence microscopy. Thereafter, animals were sacrificed with an overdose of anaesthetics and the implants with the surrounding tissue were excised for further histological and immunohistochemical analyses.

\section{Histology and immunohistochemistry}

Formalin-fixed specimens of ad-MVF-seeded nonimplanted and implanted scaffolds were embedded in paraffin-wax and cut into $3 \mu \mathrm{m}$-thick sections. Sections were stained with haematoxylin and eosin (HE), sirius red and antibodies against the apoptosis marker cleaved caspase (Casp)-3, the proliferation marker Ki67, the endothelial marker CD31 and the fluorescence marker GFP, as described previously in detail (Laschke et al., 2019a). Sections were analysed using a BX60 microscope (Olympus, Hamburg, Germany).

Collagen content of implanted ad-MVF-seeded scaffolds, as assessed by means of the reddish appearance of mature sirius-red-stained collagen type I fibres under polarised-light microscopy in relation to normal skin, was measured in 4 ROIs of each sample using the imaging software cellSens Dimension 1.11 (Olympus) (Frueh et al., 2017a). Additional quantitative analyses included the determination of the fractions of Casp $-3^{+}$and $\mathrm{Ki} 67^{+}$ cells (given in percentage) in randomly selected ad-MVF, including at least 100 endothelial and perivascular cells per sample. Moreover, the density of all $\mathrm{CD}_{3} 1^{+}$microvessels (given in $\mathrm{mm}^{-2}$ ) and the fraction of $\mathrm{CD} 31^{+} / \mathrm{GFP}^{+}$microvessels of all CD $31^{+}$ microvessels (given in percentage) were assessed in the centre and border zones of ad-MVF-seeded implants.

\section{Statistical analysis}

Data were tested for normal distribution and equal variance. In case of parametric distribution of the data, differences between the groups were analysed by unpaired Student's $t$-test. In case of non-
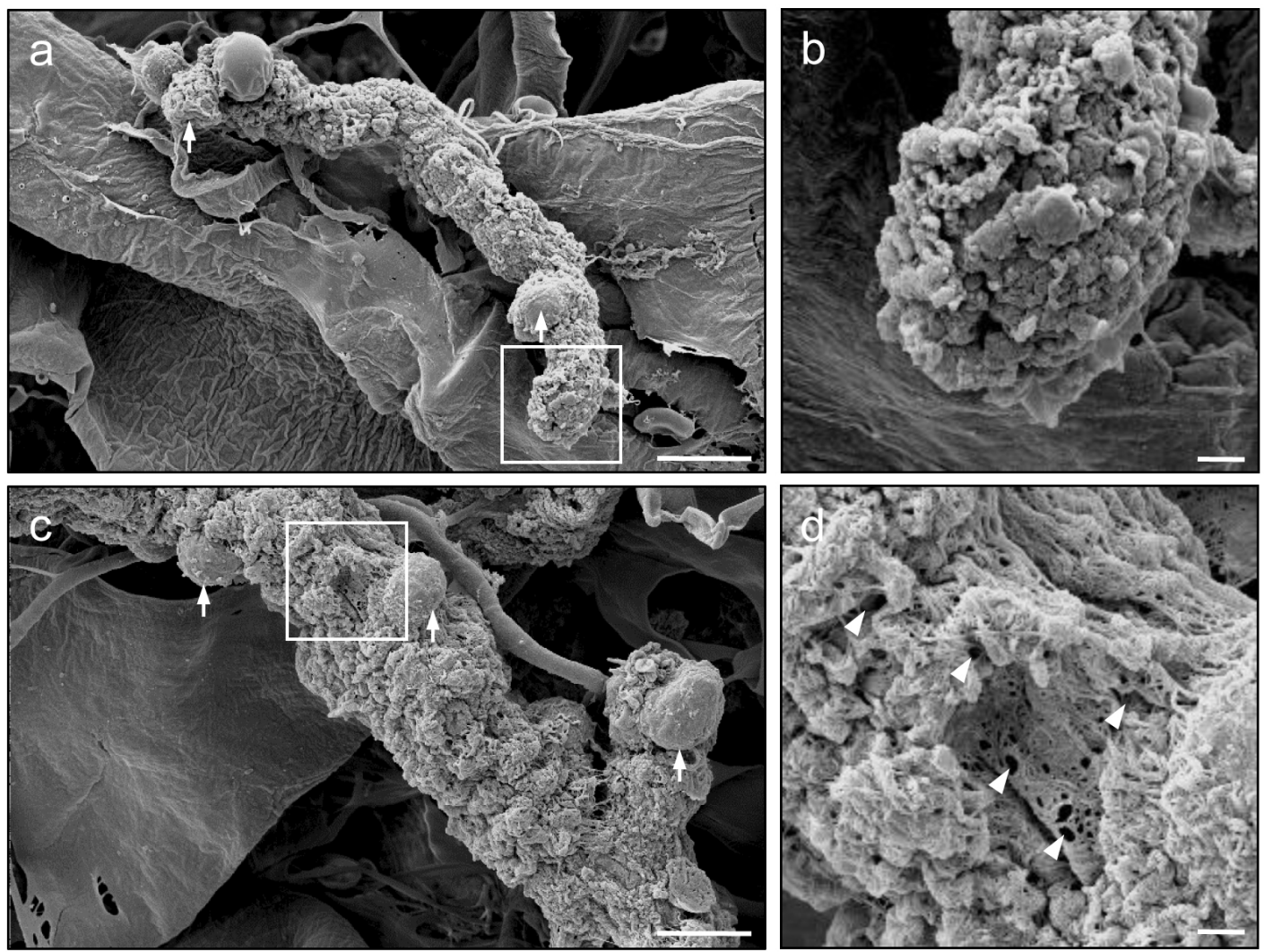

Fig. 2. Morphology of ad-MVF. (a-d) Scanning electron microscopy of ad-MVF directly after their seeding on to collagen-glycosaminoglycan matrices. ad-MVF were cultivated for $24 \mathrm{~h}$ in $4{ }^{\circ} \mathrm{C}$ UW solution supplemented with $(\mathbf{a}, \mathbf{b})$ vehicle or $(\mathbf{c}, \mathbf{d})$ high glucose. Arrows = pericytes; arrowheads = nanopores. $(\mathbf{b}, \mathbf{d})$ Higher magnification (scale bar: $1.4 \mu \mathrm{m}$ ) of inserts in a and c (scale bar: $11 \mu \mathrm{m})$. 
parametric distribution of the data, differences were assessed by Mann-Whitney rank sum test (SigmaPlot 11.0; Jandel Corporation, San Rafael, CA, USA). All values are given as mean \pm standard error of the mean (SEM). Statistical significance was accepted for a value of $p<0.05$.

\section{Results}

\section{ad-MVF protein expression}

In a first set of experiments, a proteome profiler mouse angiogenesis array was performed to analyse the expression of pro- and anti-angiogenic factors in cultivated ad-MVF. This array revealed a difference in protein expression pattern between vehicle- and highglucose-treated ad-MVF (Table 1). In comparison to controls, the most increased protein levels were detected in high-glucose-exposed ad-MVF for the pro-angiogenic factors endoglin, interleukin (IL)$1 \beta$ and monocyte chemoattractant protein (MCP)1 . In contrast, the pro-angiogenic factor matrix metalloproteinase (MMP)-8 and its counterpart tissue inhibitor of matrix metalloproteinase (TIMP)-1 were the most downregulated proteins (Table 1).

\section{Morphology of ad-MVF}

Ad-MVF were seeded on to collagenglycosaminoglycan scaffolds directly after the 24 h-cultivation period and analysed by means
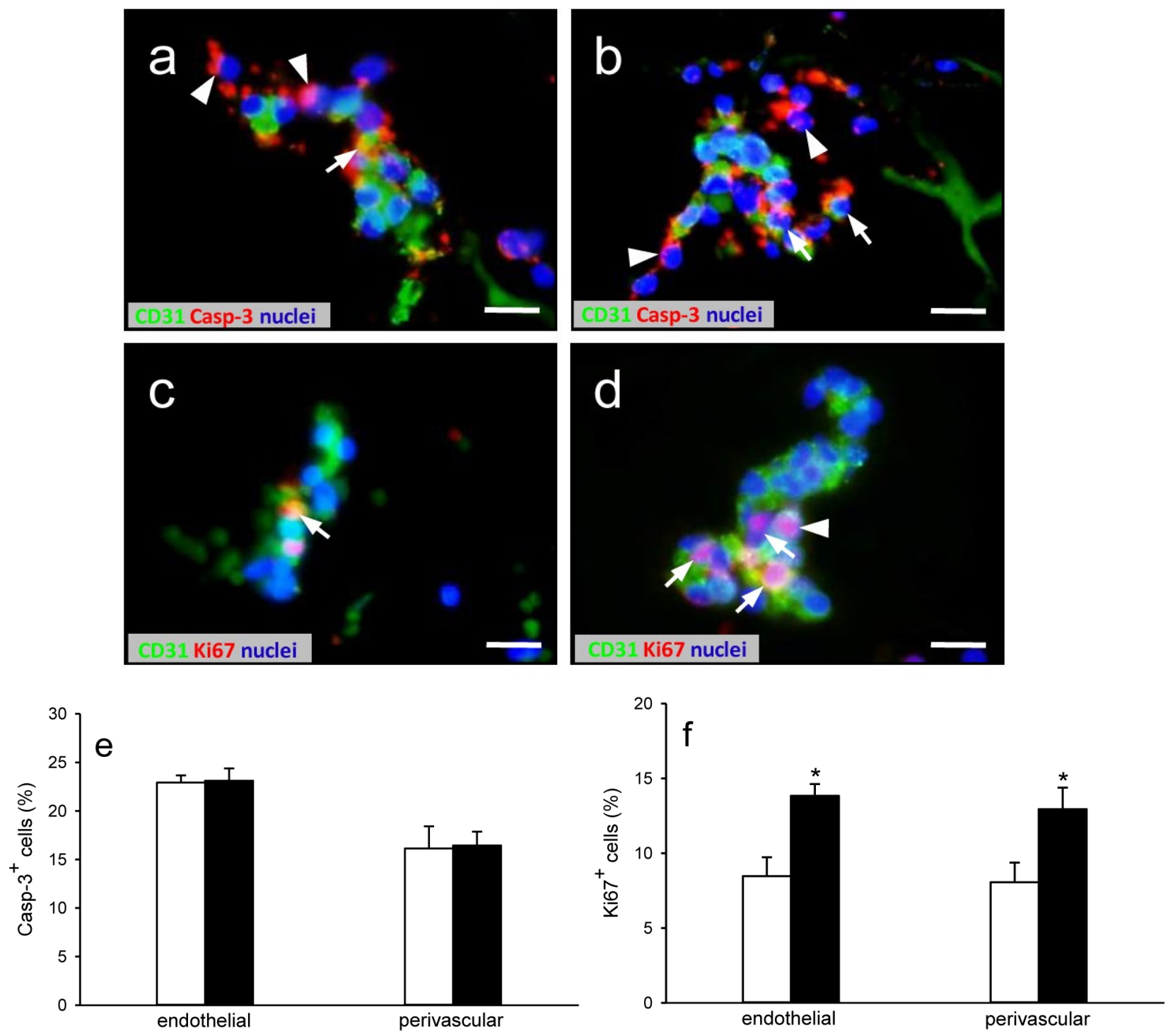

Fig. 3. Viability and proliferation of ad-MVF. (a-d) Fluorescence microscopy of ad-MVF directly after their seeding on to collagen-glycosaminoglycan matrices. ad-MVF were cultivated for $24 \mathrm{~h}$ in $4{ }^{\circ} \mathrm{C}$ UW solution supplemented with $(\mathbf{a}, \mathbf{c})$ vehicle or $(\mathbf{b}, \mathbf{d})$ high glucose. $(\mathbf{a}-\mathbf{d})$ Staining was performed with Hoechst 33342 (blue) for the detection of cell nuclei and an antibody against CD31 (green) for the identification of endothelial cells in combination with an antibody against $(\mathbf{a}, \mathbf{b})$ Casp-3 (red) for the labelling of apoptotic cells or (c,d) against Ki67 (red) for the labelling of proliferating cells. Arrows = marker-positive endothelial cells; arrowheads = marker-positive perivascular cells. Scale bars: $10 \mu \mathrm{m}$. Percentage of (e) Casp- $3^{+}$cells and (f) $\mathrm{Ki}^{+} 7^{+}$cells within ad-MVF, which were cultivated for $24 \mathrm{~h}$ in $4{ }^{\circ} \mathrm{C} \mathrm{UW}$ solution supplemented with vehicle (white bars, $n=4$ ) or high glucose (black bars, $n=4$ ). Mean \pm SEM; ${ }^{*} p<0.05$ vs. vehicle. 
of scanning electron microscopy to assess their morphology (Fig. 2a-d). Both high-glucose- and vehicle-exposed ad-MVF exhibited a comparable vessel morphology, with attached stabilising pericytes (Fig. 2a,c). However, higher magnifications revealed multiple nanopores in high-glucose-exposed ad-MVF, which could not be observed in vehicletreated controls, indicating a beginning disintegration of their vessel walls (Fig. $2 \mathbf{b}, \mathbf{d}$ ).

\section{Viability and proliferation of ad-MVF}

Immunohistochemical analyses showed a comparable fraction of $\mathrm{Casp}-3^{+}$apoptotic endothelial and perivascular cells in vehicle- and high-glucoseexposed ad-MVF (Fig. 3a,b,e). In contrast, highglucose-exposed ad-MVF exhibited a significantly higher number of $\mathrm{Ki} 67^{+}$proliferating endothelial and perivascular cells when compared to controls (Fig. 3c,d,f).

\section{In vivo vascularisation capacity of ad-MVF}

To assess their in vivo vascularisation capacity, $\mathrm{GFP}^{+}$vehicle- and high-glucose-exposed ad-MVF were seeded on to collagen-glycosaminoglycan scaffolds, which were implanted into the dorsal skinfold chambers of $\mathrm{GFP}^{-}$wild-type recipient mice. Repetitive intravital fluorescent microscopy analyses of the implants revealed that ad-MVF of both groups were able to rapidly reassemble into microvascular networks (Fig. 4a-j). These networks also developed interconnections to the surrounding host microvasculature. Accordingly, there were no significant differences in the fraction of perfused ROIs between the two groups throughout the 14 d-observation period (Fig. 4k). However, more detailed analyses of the newly developing networks showed a significantly higher functional microvessel density in scaffolds seeded with high-glucoseexposed ad-MVF between day 6 and 14 when compared to controls (Fig. 4l). In addition, individual microvessels within these scaffolds presented with a significantly smaller diameter and higher centreline $\mathrm{RBC}$ velocity at later observation time points (Fig. 5a-d). Accordingly, the calculated wall shear rate of these vessels was also significantly higher when compared to controls (Fig. 5e). These findings
$0 d$

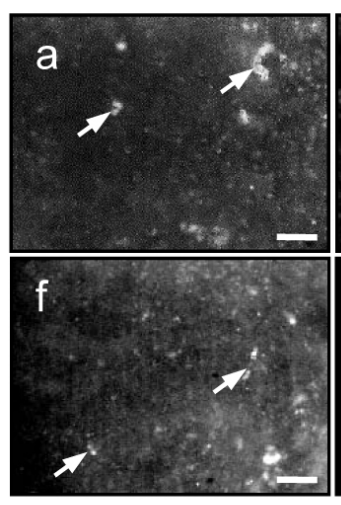

$3 d$

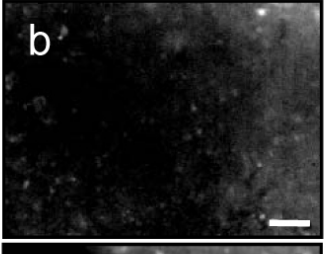

$6 d$

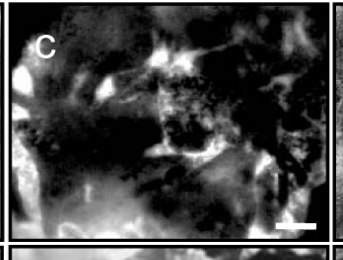

$10 \mathrm{~d}$

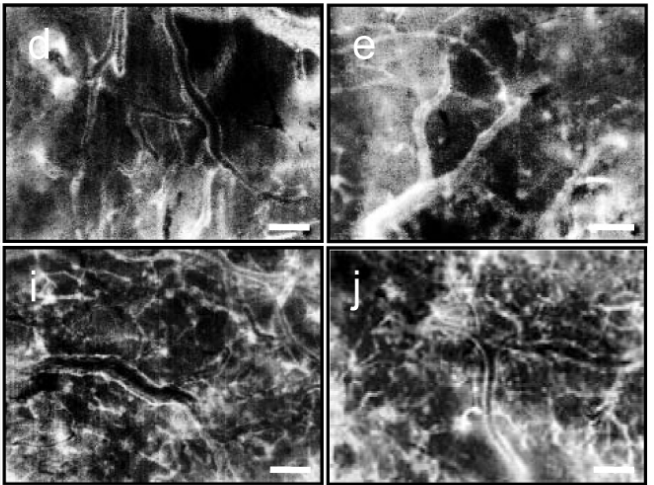

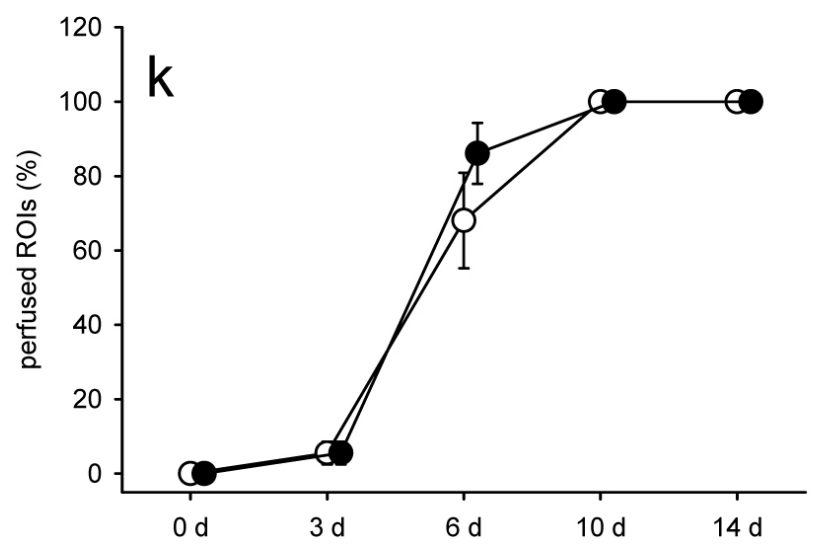

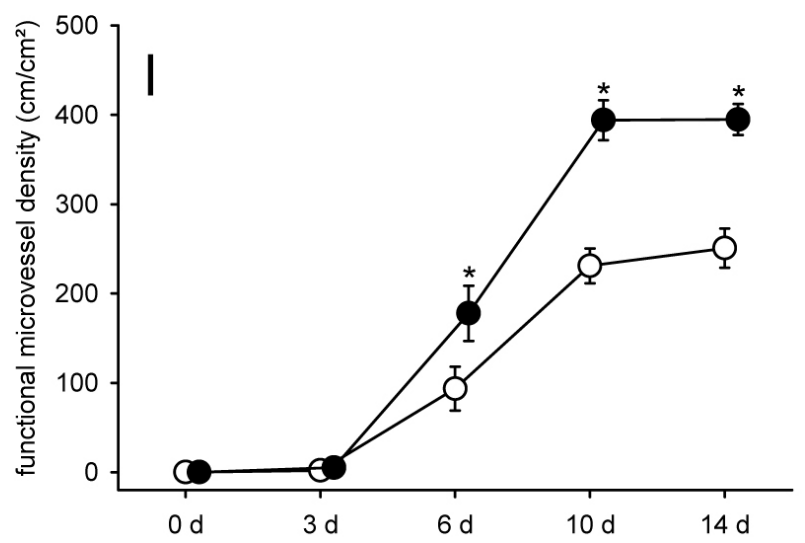

Fig. 4. In vivo vascularisation capacity of ad-MVF. (a-j) Intravital fluorescence microscopy (blue-light epiillumination with contrast enhancement by $5 \%$ FITC-labelled dextran 150,000 injected intravenous) of adMVF-seeded collagen-glycosaminoglycan scaffolds on day $0,3,6,10$ and 14 after implantation into the dorsal skinfold chamber of C57BL/6 mice. ad-MVF were cultivated for $24 \mathrm{~h}$ in $4{ }^{\circ} \mathrm{C} \mathrm{UW}$ solution supplemented with (a-e) vehicle or $(\mathbf{f}-\mathbf{j})$ high glucose. Arrows $=G P^{+}$ad-MVF. Scale bars: $85 \mu \mathrm{m}$. (k) Percentage of perfused ROIs and (1) functional microvessel density $\left(\mathrm{cm} / \mathrm{cm}^{2}\right)$ of ad-MVF-seeded collagen-glycosaminoglycan scaffolds directly $(0 \mathrm{~d})$ as well as 3, 6, 10 and $14 \mathrm{~d}$ after implantation into dorsal skinfold chambers, as assessed by intravital fluorescence microscopy. ad-MVF were cultivated for $24 \mathrm{~h}$ in $4{ }^{\circ} \mathrm{C} \mathrm{UW}$ solution supplemented with vehicle (white circles, $n=9$ ) or high glucose (black circles, $n=9$ ). Mean $\pm \mathrm{SEM} ;{ }^{*} p<0.05$ vs. vehicle. 

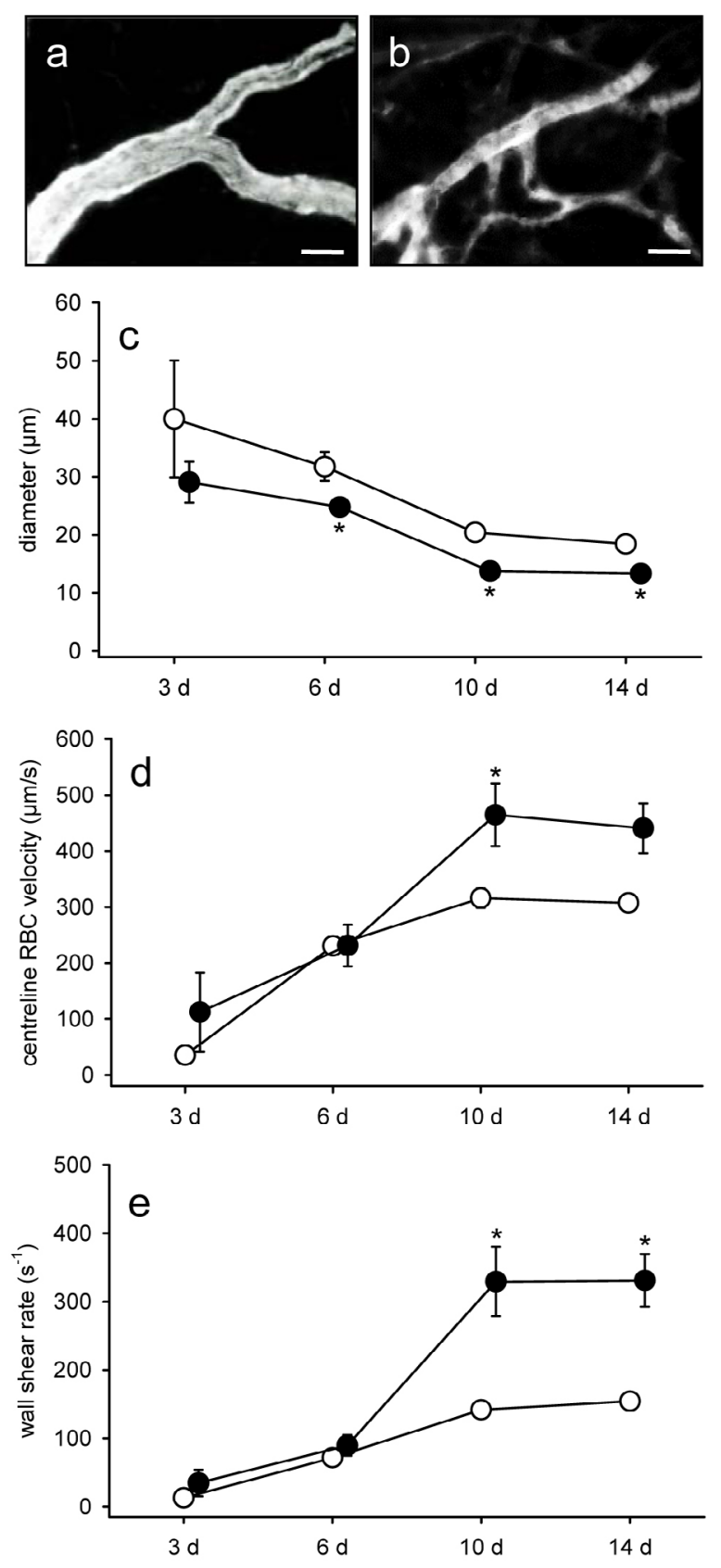

Fig. 5. Vascular maturation and remodelling of adMVF. (a,b) Intravital fluorescence microscopy (bluelight epi-illumination with contrast enhancement by $5 \%$ FITC-labelled dextran 150,000 injected intravenous) of individual microvessels within adMVF-seeded collagen-glycosaminoglycan scaffolds on day 14 after implantation into the dorsal skinfold chamber of C57BL/6 mice. ad-MVF were cultivated for $24 \mathrm{~h}$ in $4{ }^{\circ} \mathrm{C}$ UW solution supplemented with (a) vehicle or (b) high glucose. Scale bars: $20 \mu \mathrm{m}$. (c) Diameter $(\mu \mathrm{m}),($ d) centreline RBC velocity $(\mu \mathrm{m} / \mathrm{s})$ and $(\mathbf{e})$ wall shear rate $\left(\mathrm{s}^{-1}\right)$ of individual microvessels within ad-MVF-seeded collagenglycosaminoglycan scaffolds 3, 6, 10 and $14 \mathrm{~d}$ after implantation into dorsal skinfold chambers, as assessed by intravital fluorescence microscopy. adMVF were cultivated for $24 \mathrm{~h}$ in $4{ }^{\circ} \mathrm{C}$ UW solution supplemented with vehicle (white circles, $n=9$ ) or high glucose (black circles, $n=9$ ). Mean \pm SEM; ${ }^{*} p<0.05$ vs. vehicle. indicated an improved vascularisation as well as vascular maturation and remodelling of the scaffolds with the high-glucose-exposed ad-MVF.

At the end of the in vivo experiments, ad-MVFseeded scaffolds were additionally analysed by means of histology and immunohistochemistry. HE-stained sections showed a denser granulation tissue within the pores and the surrounding host tissue of implants seeded with high-glucose-exposed ad-MVF (Fig. 6a,b). These scaffolds also exhibited a significantly higher microvessel density in their border zones when compared to controls (Fig. 6c,f,i). In contrast, the microvessel density in the centre of the implants was comparable in both groups (Fig. 6i). Moreover, they presented with a comparably high fraction of $>80 \% \mathrm{GFP}^{+}$microvessels in their border and centre zones (Fig. 6d,e, $, \mathbf{g}, \mathbf{h}, \mathbf{j}$ ). Finally, the analysis of sirius-red-stained sections revealed a tendency towards a higher total collagen ratio in scaffolds seeded with high-glucose-exposed ad-MVF, indicating an improved tissue incorporation when compared to controls (Fig. 6k-n).

\section{Discussion}

The present study demonstrated that exposure of cultivated ad-MVF to high glucose concentrations promoted their proliferation and in vivo network formation. Hence, this novel approach may improve the outcome of future therapeutic interventions using ad-MVF as vascularisation units. The shortterm cultivation of ad-MVF may be particularly recommended in clinical cases in which freshly isolated ad-MVF cannot be directly retransferred into patients due to prolonged or multi-step surgical procedures. For this purpose, ad-MVF should be cultivated at $4{ }^{\circ} \mathrm{C}$ in UW solution to prevent their agglomeration and to ensure xeno-free culture conditions according to good manufacturing practices (Laschke et al., 2019b). Those novel results indicated that these conditions can be further optimised by adding $30 \mathrm{mM}$ glucose to the UW solution, which is, in contrast to the use of recombinant growth factors, a cost-effective and, from a regulatory point of view, harmless approach to effectively increase the angiogenic activity of ad-MVF.

The pro-angiogenic effect of high glucose is not a novel finding but well known from studies focusing on the mechanisms underlying diabetes-induced aberrant angiogenesis (Betts-Obregon et al., 2016; Fernando et al., 2018; Qiu et al., 2018; Shi et al., 2019). Such studies are often performed with endothelial cell monocultures that are exposed to different glucose concentrations. In contrast, in the present study, ad-MVF were used with an intact vessel morphology and physiological cellular composition, including microvessel-attached pericytes. Hence, these fully functional vessel segments may provide more realistic conditions to study the effects of high 

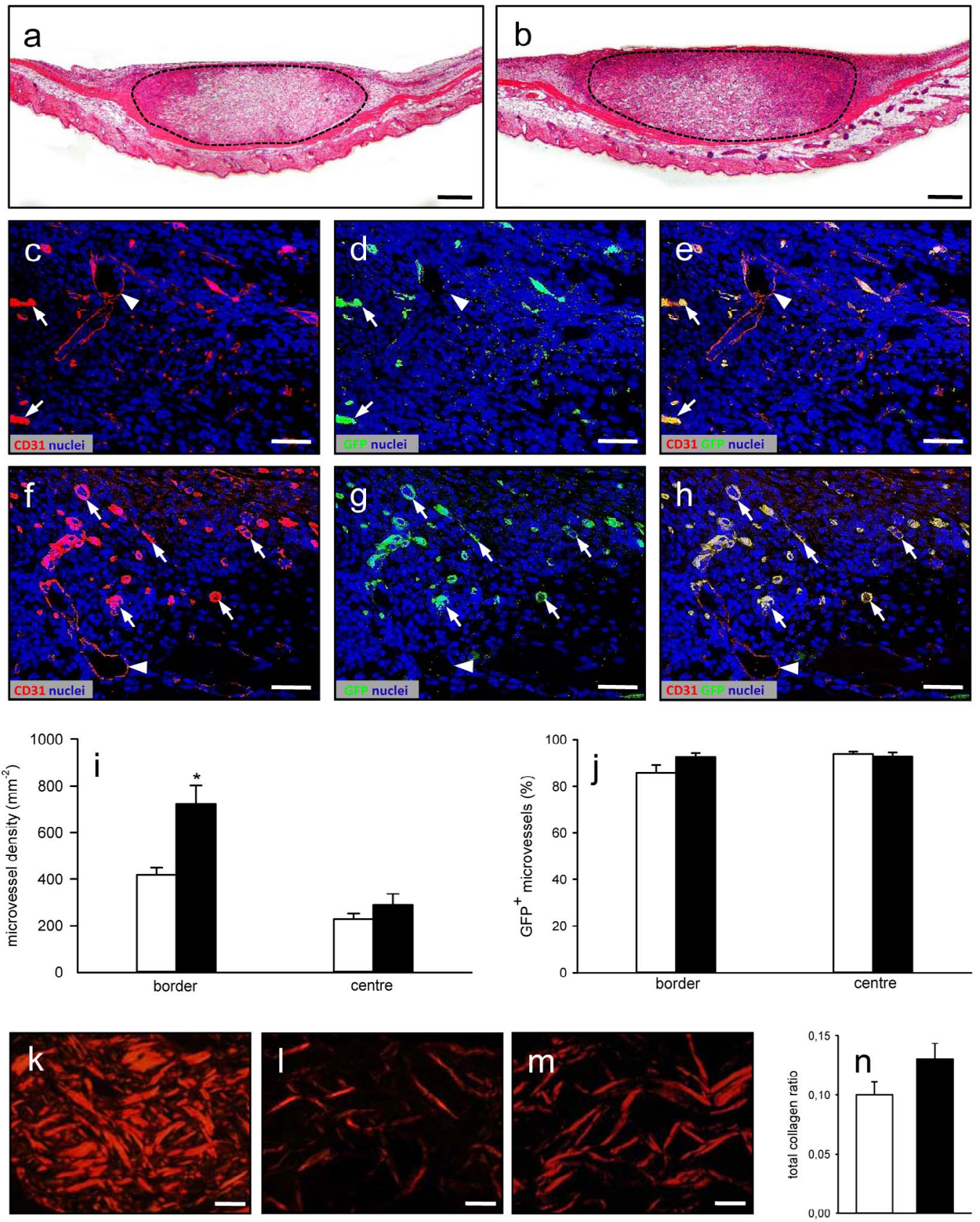

Fig. 6. Final vascularisation and incorporation of ad-MVF-seeded scaffolds. (a,b) HE-stained sections of ad-MVF-seeded collagen-glycosaminoglycan scaffolds (borders marked by broken line) on day 14 after implantation into the dorsal skinfold chamber of C57BL/6 mice. ad-MVF were cultivated for $24 \mathrm{~h}$ in $4{ }^{\circ} \mathrm{C} \mathrm{UW}$ solution supplemented with (a) vehicle or (b) high glucose. Scale bars: $350 \mu \mathrm{m}$. (c-h) Immunohistochemically stained microvessels in the border of ad-MVF-seeded collagen-glycosaminoglycan scaffolds on day 14 after implantation into the dorsal skinfold chambers of C57BL/6 mice. ad-MVF were cultivated for $24 \mathrm{~h}$ in $4^{\circ} \mathrm{C} \mathrm{UW}$ solution supplemented with (c-e) vehicle or (f-h) high glucose. (c-h) Staining was performed with Hoechst 33342 to identify cell nuclei (blue), (c,f) an antibody against CD31 for the detection of endothelial cells (red) and $(\mathbf{d}, \mathbf{g})$ an antibody against GFP (green). (e,h) Merge of $\mathbf{c}, \mathbf{d}$ and $\mathbf{f}, \mathbf{g}$. Arrows $=\mathrm{CD} 31^{+} / \mathrm{GFP}^{+}$microvessels; arrowheads $=\mathrm{CD}^{+} / \mathrm{GFP}^{-}$microvessels. Scale bars: $25 \mu \mathrm{m}$. (i) Microvessel density $\left(\mathrm{mm}^{-2}\right)$ and (j) $\mathrm{GFP}^{+}$ microvessels percentage in the centre and border zones of ad-MVF-seeded collagen-glycosaminoglycan scaffolds $14 \mathrm{~d}$ after implantation into the dorsal skinfold chamber, as assessed by immunohistochemical analysis. ad-MVF were cultivated for $24 \mathrm{~h}$ in $4^{\circ} \mathrm{C} \mathrm{UW}$ solution supplemented with vehicle (white bars, $n=9$ ) or high glucose (black bars, $n=9$ ). Mean $\pm \mathrm{SEM}^{*}{ }^{*}<0.05$ vs. vehicle. Sirius-red-stained sections of $(\mathbf{k})$ normal skin as well as $(\mathbf{l}, \mathbf{m})$ ad-MVF-seeded collagen-glycosaminoglycan scaffolds on day 14 after implantation into the dorsal skinfold chamber of C57BL/6 mice. ad-MVF were cultivated for $24 \mathrm{~h}$ in $4{ }^{\circ} \mathrm{C} \mathrm{UW}$ solution supplemented with (1) vehicle or ( $\mathbf{m})$ high glucose. Scale bars: $30 \mu \mathrm{m}$. (n) Total collagen ratio in ad-MVFseeded collagen-glycosaminoglycan scaffolds $14 \mathrm{~d}$ after implantation into the dorsal skinfold chamber, as assessed by histological analysis. ad-MVF were cultivated for $24 \mathrm{~h}$ in $4{ }^{\circ} \mathrm{C}$ UW solution supplemented with vehicle (white bar, $n=9$ ) or high glucose (black bar, $n=9$ ). Mean \pm SEM. 
glucose on microvessels and, thus, may also be recommended as explants for future in vitro studies in diabetes research. In line with this view, not only was the stimulating effect of glucose on endothelial cell proliferation (Fernando et al., 2018; Qiu et al., 2018) confirmed, but also larger numbers of $\mathrm{Ki}^{+} 7^{+}$ proliferating perivascular cells were detected in high-glucose-exposed ad-MVF when compared to vehicle-exposed controls. This indicated that vessel-wall-stabilising cells crucially contribute to diabetes-related microvasculopathy. Moreover, scanning electron microscopy analyses revealed that high-glucose-exposed ad-MVF exhibited multiple nanopores. On the one hand, these nanopores may result from glucose-induced cellular damage and beginning necrosis. On the other hand, they may be interpreted as an early sign of angiogenesis, which is typically associated with an increased vascular permeability, degradation of the basal lamina and disintegration of the vessel wall (Díaz-Flores et al., 2017). When getting larger, these nanopores may also represent the morphological correlate to the haemorrhages that are clinically observed in diabetic retinopathy (Murugesan et al., 2015). Diabetes-induced microvascular abnormalities additionally include microaneurysms, exudates and microthromboses (Murugesan et al., 2015), indicating an impaired vessel functionality. Hence, it is reasonable to assume that a longer exposure to supraphysiological glucose levels, as they occur in diabetic patients, would rather reduce the regenerative potential of ad-MVF. However, this issue needs further clarification.

A major prerequisite for the reassembly of adMVF into new microvascular networks is their growth towards each other by the process of sprouting angiogenesis (Nunes et al., 2010). This process is initiated and driven by the complex interplay of multiple pro- and anti-angiogenic factors (Weis and Cheresh, 2011). To study the effect of high glucose on the expression of these factors, a proteome profiler mouse angiogenesis array was performed. Of interest, an altered protein expression pattern was found in high-glucose-exposed adMVF, with endoglin, IL-1 $\beta$ and MCP-1 as the most upregulated factors when compared to vehicleexposed controls. Endoglin is a glycoprotein, which is typically upregulated in angiogenic endothelial cells, where it promotes proliferation and migration via transforming growth factor (TGF)- $\beta$ /activin receptorlike kinase (ALK)1/Smad1/5/8 signalling (Kasprzak and Adamek, 2018). IL-1 $\beta$ and MCP-1 are primarily known as pro-inflammatory cytokines, which recruit different immune cell types to sites of inflammation (Bianconi et al., 2018; Fenini et al., 2017). However, several studies have shown that they also exert proangiogenic effects, indicating a close link between inflammation and angiogenesis (Hayashi et al., 2015; Mohr et al., 2017; Sun et al., 2016). Of note, this link is a typical feature of diabetes-related angiogenic pathologies too (Capitão and Soares, 2016). In addition, a strong down-regulation of pro-angiogenic factors, such as MMP-8, was detected that did not, however, shift the analysed ad-MVF towards an antiangiogenic phenotype. This may be explained by the fact that anti-angiogenic factors, such as TIMP-1, the counterpart of MMP-8, were also down-regulated in high-glucose-exposed ad-MVF.

To assess their in vivo vascularisation capacity, vehicle- and high-glucose-exposed ad-MVF from $\mathrm{GFP}^{+}$donor mice were analysed in dorsal skinfold chambers of $\mathrm{GFP}^{-}$recipient animals. By means of this $\mathrm{GFP}^{+} / \mathrm{GFP}^{-}$cross-over design, it was demonstrated that in both groups most of the $\mathrm{GFP}^{+}$ad-MVF survived and mainly contributed to the final vascularisation of the seeded scaffolds at day 14 after implantation. However, this vascularisation was markedly improved in the group of high-glucose-treated adMVF, as indicated by a significantly higher functional microvessel density when compared to controls. This finding was in line with the pro-angiogenic effects of high glucose observed in the in vitro analyses. Besides these effects, other mechanisms may have further contributed to this positive outcome. In fact, Yoon et al. (2014) reported that high glucose conditions induce Jagged1 and suppress Notch1 expression in endothelial cells. These conditions promote the specification of endothelial cells to the tip cell phenotype, which is highly motile and guides endothelial sprouts towards growth factor stimuli (Chen et al., 2019). Accordingly, Yoon et al. (2014) additionally detected an increased angiogenic sprouting and branching of glucose-exposed endothelial cell/smooth muscle cell spheroids, resulting in a higher density of newly developing microvascular networks. Furthermore, high shear forces, as assessed in the present study in individual microvessels of the high glucose group, stimulate vascular sprouting (Galie et al., 2014).

\section{Conclusion}

Exposure of cultivated ad-MVF to high glucose concentrations resulted to be a promising approach to improve their in vivo vascularisation capacity. A rapid and sufficient vascularisation is of pivotal importance for the incorporation of biomaterials into the surrounding host tissue (Reichel et al., 2015). In the present study, this was reflected by a denser granulation tissue and a higher collagen content within the scaffolds seeded with high-glucoseexposed ad-MVF when compared to controls. In addition, survival and long-term function of implanted tissue constructs was crucially determined by an adequate blood supply (Laschke et al., 2006). Hence, high-glucose-exposed ad-MVF may serve as versatile vascularisation units to use in a wide range of future applications in the fields of implant research, tissue engineering and regenerative medicine. 


\section{Acknowledgements}

We are grateful for the excellent technical assistance of Janine Becker, Caroline Bickelmann and Julia Parakenings (Institute for Clinical and Experimental Surgery, Saarland University, Homburg/Saar, Germany). This study was funded by a grant of the Deutsche Forschungsgemeinschaft (DFG - German Research Foundation) - LA 2682/7-1.

\section{References}

Aouiss A, Anka Idrissi D, Kabine M, Zaid Y (2019) Update of inflammatory proliferative retinopathy: ischemia, hypoxia and angiogenesis. Curr Res Transl Med 67: 62-71.

Betts-Obregon BS, Vellanki S, Buikema J, Tsin AT, Wright K (2016) Effect of glucose on retinal endothelial cell viability and VEGF secretion. HSOA J Cell Biol Cell Metabol 3: 008. DOI: 10.24966/CBCM1943/100008.

Bianconi V, Sahebkar A, Atkin SL, Pirro M (2018) The regulation and importance of monocyte chemoattractant protein-1. Curr Opin Hematol 25: 44-51.

Capitão M, Soares R (2016) Angiogenesis and inflammation crosstalk in diabetic retinopathy. J Cell Biochem 117: 2443-2453.

Chen W, Xia P, Wang H, Tu J, Liang X, Zhang X, Li L (2019) The endothelial tip-stalk cell selection and shuffling during angiogenesis. J Cell Commun Signal 13: 291-301.

Díaz-Flores L, Gutiérrez R, García-Suárez MP, Sáez FJ, Gutiérrez E, Valladares F, Carrasco JL, DíazFlores L Jr, Madrid JF (2017) Morphofunctional basis of the different types of angiogenesis and formation of postnatal angiogenesis-related secondary structures. Histol Histopathol 32: 1239-1279.

Fenini G, Contassot E, French LE (2017) Potential of IL-1, IL-18 and inflammasome inhibition for the treatment of inflammatory skin diseases. Front Pharmacol 8: 278. DOI: 10.3389/fphar.2017.00278.

Fernando KHN, Yang HW, Jiang Y, Jeon YJ, Ryu B (2018) Diphlorethohydroxycarmalol isolated from Ishige okamurae represses high glucose-induced angiogenesis in vitro and in vivo. Mar Drugs 16. pii: E375. DOI: 10.3390/md16100375.

Frueh FS, Später T, Lindenblatt N, Calcagni M, Giovanoli P, Scheuer C, Menger MD, Laschke MW (2017a) Adipose tissue-derived microvascular fragments improve vascularization, lymphangiogenesis, and integration of dermal skin substitutes. J Invest Dermatol 137: 217-227.

Frueh FS, Später T, Scheuer C, Menger MD, Laschke MW (2017b) Isolation of murine adipose tissuederived microvascular fragments as vascularization units for tissue engineering. J Vis Exp 122. DOI: $10.3791 / 55721$.
Galie PA, Nguyen DH, Choi CK, Cohen DM, Janmey PA, Chen CS (2014) Fluid shear stress threshold regulates angiogenic sprouting. Proc Natl Acad Sci U S A 111: 7968-7973.

Hayashi Y, Murakami M, Kawamura R, Ishizaka R, Fukuta O, Nakashima M (2015) CXCL14 and MCP1 are potent trophic factors associated with cell migration and angiogenesis leading to higher regenerative potential of dental pulp side population cells. Stem Cell Res Ther 6: 111. DOI: 10.1186/s13287015-0088-z.

Karschnia P, Scheuer C, Heß A, Später T, Menger MD, Laschke MW (2018) Erythropoietin promotes network formation of transplanted adipose tissuederived microvascular fragments. Eur Cell Mater 35: 268-280.

Kasprzak A, Adamek A (2018) Role of endoglin (CD105) in the progression of hepatocellular carcinoma and anti-angiogenic therapy. Int J Mol Sci 19. pii: E3887. DOI: 10.3390/ijms19123887.

Laschke MW, Harder Y, Amon M, Martin I, Farhadi J, Ring A, Torio-Padron N, Schramm R, Rücker M, Junker D, Häufel JM, Carvalho C, Heberer M, Germann G, Vollmar B, Menger MD (2006) Angiogenesis in tissue engineering: breathing life into constructed tissue substitutes. Tissue Eng 12: 2093-2104.

Laschke MW, Kleer S, Scheuer C, Schuler S, Garcia P, Eglin D, Alini M, Menger MD (2012) Vascularisation of porous scaffolds is improved by incorporation of adipose tissue-derived microvascular fragments. Eur Cell Mater 24: 266-277.

Laschke MW, Menger MD (2015) Adipose tissue-derived microvascular fragments: natural vascularization units for regenerative medicine. Trends Biotechnol 33: 442-448.

Laschke MW, Karschnia P, Scheuer C, Heß A, Metzger W, Menger MD (2018) Effects of cryopreservation on adipose tissue-derived microvascular fragments. J Tissue Eng Regen Med 12: 1020-1030.

Laschke MW, Heß A, Scheuer C, Karschnia P, Menger MD (2019a) Subnormothermic short-term cultivation improves the vascularization capacity of adipose tissue-derived microvascular fragments. J Tissue Eng Regen Med 13: 131-142.

Laschke MW, Heß A, Scheuer C, Karschnia P, Kontaxi E, Menger MD (2019b) University of Wisconsin solution for the xeno-free storage of adipose tissue-derived microvascular fragments. Regen Med 14: 681-691.

Madri JA, Williams SK (1983) Capillary endothelial cell cultures: phenotypic modulation by matrix components. J Cell Biol 97: 153-165.

McDaniel JS, Pilia M, Ward CL, Pollot BE, Rathbone CR (2014) Characterization and multilineage potential of cells derived from isolated microvascular fragments. J Surg Res 192: 214-222.

Mohr T, Haudek-Prinz V, Slany A, Grillari J, Micksche M, Gerner C (2017) Proteome profiling 
in IL-1 $\beta$ and VEGF-activated human umbilical vein endothelial cells delineates the interlink between inflammation and angiogenesis. PLoS One 12: e0179065. DOI: 10.1371/journal.pone.0179065.

Murugesan N, Üstunkaya T, Feener EP (2015) Thrombosis and hemorrhage in diabetic retinopathy: a perspective from an inflammatory standpoint. Semin Thromb Hemost 41: 659-664.

Nunes SS, Greer KA, Stiening CM, Chen HY, Kidd KR, Schwartz MA, Sullivan CJ, Rekapally H, Hoying JB (2010) Implanted microvessels progress through distinct neovascularization phenotypes. Microvasc Res 79: 10-20.

Okabe M, Ikawa M, Kominami K, Nakanishi T, Nishimune Y (1997) 'Green mice' as a source of ubiquitous green cells. FEBS Lett 407: 313-319.

Qiu F, Tong H, Wang Y, Tao J, Wang H, Chen L (2018) Recombinant human maspin inhibits high glucose-induced oxidative stress and angiogenesis of human retinal microvascular endothelial cells via PI3K/AKT pathway. Mol Cell Biochem 446: 127-136.

Reichel CA, Hessenauer ME, Pflieger K, Rehberg M, Kanse SM, Zahler S, Krombach F, Berghaus A, Strieth S (2015) Components of the plasminogen activation system promote engraftment of porous polyethylene biomaterial via common and distinct effects. PLoS One 10: e0116883. DOI: 10.1371/journal. pone.0116883.

Sato N, Nariuchi H, Tsuruoka N, Nishihara T, Beitz JG, Calabresi P, Frackelton AR Jr (1990) Actions of TNF and IFN-gamma on angiogenesis in vitro. J Invest Dermatol 95: 85S-89S.

Sato N, Beitz JG, Kato J, Yamamoto M, Clark JW, Calabresi P, Raymond A, Frackelton AR Jr (1993) Platelet-derived growth factor indirectly stimulates angiogenesis in vitro. Am J Pathol 142: 1119-1130.

Shepherd BR, Hoying JB, Williams SK (2007) Microvascular transplantation after acute myocardial infarction. Tissue Eng 13: 2871-2879.

Shi Y, Chen C, Xu Y, Liu Y, Zhang H, Liu Y (2019) LncRNA FENDRR promotes high-glucose-induced proliferation and angiogenesis of human retinal endothelial cells. Biosci Biotechnol Biochem 83: 869875.

Später T, Frueh FS, Nickels RM, Menger MD, Laschke MW (2018) Prevascularization of collagenglycosaminoglycan scaffolds: stromal vascular fraction versus adipose tissue-derived microvascular fragments. J Biol Eng 12: 24. DOI: 10.1186/s13036-0180118-3.

Sun J, Chen J, Cao J, Li T, Zhuang S, Jiang $X$ (2016) IL-1 $\beta$-stimulated $\beta$-catenin up-regulation promotes angiogenesis in human lung-derived mesenchymal stromal cells through a NF- $\kappa \mathrm{B}-$ dependent microRNA-433 induction. Oncotarget 7: 59429-59440.

Weis SM, Cheresh DA (2011) Tumor angiogenesis: molecular pathways and therapeutic targets. Nat Med 17: 1359-1370.

Williams SK, Devenny JJ, Bitensky MW (1981) Micropinocytic ingestion of glycosylated albumin by isolated microvessels: possible role in pathogenesis of diabetic microangiopathy. Proc Natl Acad Sci U S A 78: 2393-2397.

Yoon $\mathrm{CH}$, Choi YE, Koh SJ, Choi JI, Park YB, Kim HS (2014) High glucose-induced jagged 1 in endothelial cells disturbs notch signaling for angiogenesis: a novel mechanism of diabetic vasculopathy. J Mol Cell Cardiol 69: 52-66.

Zent R, Pozzi A (2007) Angiogenesis in diabetic nephropathy. Semin Nephrol 27: 161-171.

\section{Discussion with Reviewers}

Daniel Schmauss: How did you choose the glucose concentration used? Could higher glucose concentrations have an even more pronounced effect? Authors: The glucose concentration was chosen based on previous in vitro studies focusing on the angiogenic effects of glucose on endothelial cells (Betts-Obregon et al., 2016; Fernando et al., 2018; Qiu et al., 2018). In these studies, a concentration of $30 \mathrm{mM}$ glucose is typically used to mimic hyperglycaemic conditions, as they occur in diabetic patients.

Daniel Schmauss: Do you think the nanopores in the vessel wall, described in the high-glucose group, might have a negative impact on the performance of the used ad-MVF if they would get larger? Or is this improbable given the short-term exposure to high glucose concentrations?

Authors: The observed nanopores may be interpreted in different ways. On the one hand, they may result from glucose-induced cellular damage and beginning necrosis. In this case, they would probably have a negative impact on the in vivo performance of the ad-MVF. On the other hand, they may be interpreted as an early sign of angiogenesis, which is typically associated with an increased vascular permeability, degradation of the basal lamina and disintegration of the vessel wall (Díaz-Flores et al., 2017). If this holds true, the nanopores would have a positive impact when getting larger during glucose exposure, resulting in an accelerated in vivo reassembly of individual ad-MVF into new microvascular networks.

Heiko Sorg: Have you tested systemic glucose administration?

Authors: No, in the present study, the systemic treatment of mice with high glucose concentrations was deliberately omitted. In fact, the aim was to test a new approach for improving the in vivo vascularisation capacity of ad-MVF without affecting the physiological health status of the recipients. In clinical practice, such an approach would be much more attractive, because potential side effects of a systemic treatment could be avoided. Nonetheless, we feel that the reviewer addresses the interesting question of how ad-MVF would perform when applied in diabetic patients. Since the prevalence of diabetes is continuously increasing, being particularly 
high in older patients, who may also represent a main target group for future tissue engineering therapies, this relevant issue should be clarified in further experimental studies.

Heiko Sorg: From a clinical point of view, might liposuction be also sufficient to gain significant amounts of adMVF?

Authors: The isolation of ad-MVF from human liposuctioned fat is the desirable method for the future application of this concept in clinical practice. Currently, protocols are being developed to promote exactly this approach and, based on preliminary results, it seems possible to gain sufficient amounts of ad-MVF from this fat source.

Heiko Sorg: What would you presume would happen if exposing adMVF for more than $24 \mathrm{~h}$ to high glucose concentrations $(<72 \mathrm{~h})$ ? Might the shown effects be decreased?

Authors: We do not recommend exposing ad-MVF in vitro for more than $24 \mathrm{~h}$ to high glucose concentrations. In fact, ad-MVF lose their physiological vessel morphology when cultivated longer. Accordingly, they also exhibit an impaired in vivo vascularisation capacity (Laschke et al., 2015, additional reference). For these reasons, it is possible that the shown effects would indeed be decreased.

Heiko Sorg: The increase in collagen might also lead to fibrosis and scar formation. Can you comment on this?

Authors: Indeed, in the long run, the increased collagen formation may contribute to fibrosis and scarring. Because the present study assessed the incorporation of the scaffolds only in an early phase, i.e. $14 \mathrm{~d}$ after implantation, it is not possible to make a valid statement about the long-term performance of the implants. However, an early collagen formation was observed also within the pores of ad-MVFseeded scaffolds and not only at their interface with the surrounding host tissue. Hence, this pattern rather indicated an improved tissue ingrowth and implant incorporation, whereas a sole collagen formation around the implants would rather be a sign for scarring and scaffold fibrotic encapsulation over time.

Heiko Sorg: How do you explain the nearly explosive increase in perfused ROIs in Fig. 4 from day 3 nearly nothing to about $70-80 \%$ ?

Authors: This vascularisation pattern is typical for ad-MVF-seeded scaffolds. Due to their preserved vessel morphology, they rapidly reassemble in vivo into new microvascular networks. As soon as these networks also develop interconnections with the vessels of the surrounding host tissue, which typically occurs 3-6 d after implantation, they are completely blood-perfused within a short time. In contrast, scaffold vascularisation by angiogenic ingrowth of newly developing microvessels from the host microvasculature would be characterised by a slower but more uniform increase in perfused ROIs over time (Laschke et al., 2010, additional reference).

\section{Additional Reference}

Laschke MW, Strohe A, Menger MD, Alini M, Eglin D (2010) In vitro and in vivo evaluation of a novel nanosize hydroxyapatite particles/poly(esterurethane) composite scaffold for bone tissue engineering. Acta Biomater 6: 2020-2027.

. Laschke MW, Kleer S, Scheuer C, Eglin D, Alini M, Menger MD (2015) Pre-cultivation of adipose tissuederived microvascular fragments in porous scaffolds does not improve their in vivo vascularisation potential. Eur Cell Mater 29: 190-200.

Editor's note: The Scientific Editor responsible for this paper was Juerg Gasser. 